\title{
Leitbild Nord-Süd : Kohärenz am Beispiel Indonesien
}

Violette Ruppanner and Matthias Meyer

\section{OpenEdition}

1 Journals

Electronic version

URL: http://journals.openedition.org/sjep/1303

DOI: $10.4000 /$ sjep.1303

ISSN: 1663-9677

Publisher

Institut de hautes études internationales et du développement

Printed version

Date of publication: 1 février 1995

Number of pages: $180-185$

ISSN: $1660-5926$

\section{Electronic reference}

Violette Ruppanner und Matthias Meyer, «Leitbild Nord-Süd : Kohärenz am Beispiel Indonesien », Schweizerisches Jahrbuch für Entwicklungspolitik [Online], 14 | 1995, Online erschienen am: 05 Mai 2013, abgerufen am 08 September 2020. URL : http://journals.openedition.org/sjep/1303 ; DOI : https://doi.org/10.4000/sjep.1303 


\title{
Leitbild Nord-Süd: Kohärenz am Beispiel Indonesien
}

\author{
Violette Ruppanner /Matthias Meyer, BAWI
}

Die Wahl der Organisatoren dieses Kolloquiums fiel wohl kaum zufällig auf Indonesien. In der Tat scheint dieses Land geradezu prädestiniert, um die neuen Leitlinien des Bundes ${ }^{1}$ für eine Entwicklungspolitik der neunziger Jahre am konkreten Fall zu testen. So gehört zum Beispiel auch Indonesien zu denjenigen Entwicklungsländern, mit der die Schweiz traditionell einen Handelsbilanzüberschuss erzielt und somit zum Ausgleich des Handelsbilanzsdefizits mit den OECD-Staaten beiträgt. Auch übertrifft dieser Überschuss die jährlichen bilateralen Entwicklungshilfeleistungen der Schweiz an Indonesien um ein Vielfaches. Auf der anderen Seite gerät Indonesien immer wieder in negative Schlagzeilen, man denke dabei an die blutige Niederschlagung der ost-timoresischen Freiheitsbewegung im Jahre 1991 oder an die kürzlich erfolgte Schliessung von drei prominenten indonesischen Wochenzeitschriften, um

1. Bericht des Bundesrates über die Nord-Süd-Beziehungen der Schweiz in den 90er Jahren vom 7. März 1994 
nur diejenigen Ereignisse zu nennen, welche in der westlichen Presse ein Echo gefunden haben.

Wir möchten in diesem Aufsatz der Fragestellung des Kolloquiums folgen. In einem ersten Teil versuchen wir daher, einige reelle und mögliche Widersprüche zu analysieren, die uns als Verwalterin öffentlicher Entwicklungshilfegelder besonders am Herzen liegen. Anschliessend soll die Frage untersucht werden, wo und wie das Leitbild praktische Anwendung finden kann. Zum Schluss äussern wir uns zu möglichen Mechanismen innerhalb und ausserhalb der Verwaltung, um den Zielen des Leitbilds näher zu kommen.

\section{Widersprüche}

Bei der Vergabe von Entwicklungshilfegeldern stellt sich uns immer wieder die Frage nach der guten Regierungsführung („good governance“), dies nicht erst seit der Verabschiedung des Leitbilds. Dieser Begriff definiert die Art und Weise, wie eine Regierung optimal das Ziel der wirtschaftlichen und sozialen Entwicklung des Staates und seiner Bevölkerung durch die Schaffung geeigneter politischer und wirtschaftlicher Rahmenbedingungen verfolgt. Damit verbunden sind eine effiziente, transparente Zuteilung knapper öffentlicher Ressourcen, ein rechtlicher Rahmen (,rule of law"), eine der Bevölkerung gegenüber verantwortliche Regierung („accountability“), die Einschränkung von Willkür und Korruption, allgemeiner Zugang zu Eigentum und Kredit und der freie Fluss von Information. Indonesien ist nicht in all diesen Bereichen ein Musterknabe. Die Menschenrechte wurden oben schon erwähnt. Weitere kritische Punkte betreffen z.B. die autoritäre Regierungsführung (verschwommene Gewaltentrennung), die fast schon endemische Korruption, die in der Endphase der Regierung Suharto eher noch im Zunehmen begriffen scheint, die faktische Macht der Armee, welche einen Fünftel der Parlamentsvertreter stellt und eine Mehrzahl der Posten der Generalsekretäre und der Generaldirektoren in der Zentralverwaltung besetzt, die duale Wirtschaftsstruktur mit einem teilweise wenig effizienten staatlichen Sektor und einer wenig liberalisierten Binnenwirtschaft, denen eine dynamische Privatwirtschaft und ein wettbewerbsfähiger Exportsektor gegenüber steht. Auf den ersten Blick also Gründe genug, die eine schweizerische Entwicklungszusammenarbeit in Indonesien in Frage stellen!

Eine solche Analyse bleibt aber in unseren Augen ungenügend. Sie beschränkt sich auf eine statische Sicht der Lage. Um ein Gesamtbild zu erhalten, ist es jedoch wichtig, die zeitliche Dimension einzubeziehen. Unter diesem Blickwinkel erscheint die Situation in Indonesien weniger dramatisch, denn

- Indonesien ist erst seit dem Dogmenwechsel nach dem Fall der Berliner Mauer dem Druck der Geberländer nach mehr "good governance" ausgesetzt. Während der Zeit des Kalten Krieges lagen die Prioritäten der Länder, die Indonesien unterstützten, in einem anderen Bereich, es galt, dessen 
strategische Bedeutung zu halten und zu unterstützen.

- aus einer wirtschaftlichen Sicht kann im Fall von Indonesien von einer eigentlichen Erfolgsgeschichte gesprochen werden. Das jährliche wirtschaftliche Wachstum der letzten 25 Jahre von durchschnittlich über 6\% ermöglichte dem Land die Reduktion der absoluten Armut von 40\% der Bevölkerung im Jahre 1978 auf 15\% 1993 (die absolute Anzahl der Armen ist mit geschätzten 27-35 Millionen allerdings immer noch erdrückend hoch). Durch ein vorsichtiges makroökonomisches Management unter Einsatz institutioneller Mechanismen gelang es der indonesischen Regierung bisher, eine stabile Wirtschaftspolitik zu führen und interne (z.B. konjunkturelle Erhitzung von 1990-91) wie auch externe (z.B. Preiszerfall des Rohöls 1986-87) Schocks abzufedern. Seit bald 10 Jahren verfolgt die Regierung auch eine Politik der graduellen Liberalisierung, Deregulierung und Öffnung nach aussen, welche es dem Land ermöglichen soll, in die Reihe der asiatischen Tiger aufzusteigen.

- auch im politischen Bereich sind Anzeichen einer graduellen Lockerung vorhanden, die nicht zuletzt auch auf den wachsenden Druck der Bevölkerung nach mehr Verantwortlichkeit der Regierung und nach mehr Mitbestimmung zurückzuführen sind. Die in den achtziger Jahren begonnene Entmilitarisierung der Bürokratie und der Regierung schreitet trotz Rückschlägen (Ernennung des ehemaligen Oberbefehlshabers der Streitkräfte zum Vizepräsidenten) voran, so wurde zum Beispiel 1993 zum ersten Mal in der indonesischen Geschichte eine Zivilperson als Vorsitzender der Regierungspartei GOLKAR gewählt und es fanden erste Gespräche und Annäherungsversuche der Regierung mit Dissidenten- und Oppositionsgruppen statt (einschliesslich Ost-Timor).

Ein weiteres Thema, welches immer wieder Aufmerksamkeit findet, liegt im potentiellen Spannungsfeld zwischen dem Ziel, mit Entwicklungshilfegeldern das Wirtschaftswachstum und den Wohlstand eines Entwicklungslands zu fördern, und demjenigen der Exportförderung. Dies trifft insbesondere auf die Mischfinanzierungen zu, welche das einzige gebundene (d.h. sie dienen dem Kauf von schweizerischen Gütern und Dienstleistungen) Instrument der schweizerischen Entwicklungshilfe sind. Wir sind der Meinung, dass diese Ziele a priori nicht miteinander in Konflikt stehen, vorausgesetzt, dass ein sorgfältiger Selektionsprozess (meist durch eine internationale Ausschreibung) im Entwicklungsland und eine gewissenhafte Projektanalyse seitens der Geberinstitution und des Entwicklungslands stattgefunden hat. Im Fall von Indonesien wurde allerdings die erste Voraussetzung nicht erfüllt. Das von der Schweiz finanzierte Thermalkraftwerk Belawan in Nordsumatra wurde aufgrund der damaligen indonesischen Position, prioritäre Projekte direkt mit den internationalen Gebern zu verhandeln, bilateral ausgehandelt. Es war der indonesischen Regierung allerdings möglich, einen Preisvergleich herzustellen, da gleichzeitig konkurrentielle Offerten anderer Geber vorlagen. Im zweiten Fall, einem Rehabilitationsprojekt einer Zahnradbahn in Westsumatra, schloss die prakti- 
sche Monopolstellung des Schweizer Lieferanten eine internationale Ausschreibung aus. Auch hier war es jedoch der indonesischen Regierung möglich, anhand früherer Lieferungen dieser Firma einen Preisvergleich zu machen. Die beiden Projekte wurden von uns gründlich auf ihre entwicklungspolitische Relevanz geprüft und deren Resultate den indonesischen Behörden vorgelegt.

Zu guter Letzt möchten wir noch auf ein Problem eingehen, welches auch zwei Jahre nach dem Erdgipfel in Rio nichts an seiner Brisanz und Aktualität verloren hat. Das rasante wirtschaftliche Wachstum ist neben der Armut die Hauptursache für die wachsende Umweltbelastung und die Zerstörung der natürlichen Lebensgrundlagen in den Entwicklungsländern. Auch Indonesien bezahlt seine ungehemmte Entwicklung der letzten Jahre zur Zeit mit irreparablen Schäden an der Umwelt. Steht hier das legitime Bedürfnis nach Wachstum und mehr Wohlstand der Entwicklungsländer im Widerspruch mit einer nachhaltigen Entwicklung? Welche Aufgabe haben die Industrieländer, darunter die Schweiz, die mit ihrem Konsumverhalten und ihrem übermässigen Verbrauch an Ressourcen für einen überdurchschnittlichen Anteil der negativen Auswirkungen auf die Umwelt verantwortlich sind? Haben wir das Recht, mit dem Finger auf "schuldige" Entwicklungsländer zu zeigen und nationale Umweltmassnahmen sowie Handelsbeschränkungen zu ergreifen, welche sich wirtschaftlich und damit vielfach langfristig auch ökologisch zu Lasten der Entwicklungsländer auswirken? Wir glauben nein. Vielmehr gilt es, mit positiven Massnahmen die Entwicklungsländer in ihren Anstrengungen für eine nachhaltige Entwicklung zu unterstützen. Konkret heisst dies, dass einerseits Entwicklungshilfeprojekte auch nach umweltpolitischen Kriterien überprüft werden und anderseits entsprechende Mittel bereitgestellt werden, um die Produktions- und Verarbeitungsmethoden von Entwicklungsländern zu verbessern, die aus finanziellen und technischen Gründen noch nicht in der Lage sind, angepasste Umweltstandards zu erreichen. Im Fall des umweltpoltitisch umstrittenen Projekts Belawan bedeutete dies z.B. konkret, dass die Mischfinanzierung mit der Auflage verbunden wurde, ein Umweltmonitoring einzubauen, welches mit Geschenksmitteln aus der Schweiz finanziert wurde.

\section{Lösungen}

Anhand dieser wenigen Beispiele wird es klar, dass angesichts der Vielfalt der Akteure, die unterschiedliche - und oft gegensätzliche - Interessen vertreten, das Vermeiden von Zielkonflikten schwierig und manchmal sogar unmöglich ist und es auch keine Schulbuchlösung geben kann, sondern die Frage von Fall zu Fall angegangen werden muss. Das Beispiel der Exportrisikogarantie (ERG), welche innenpolitisch motiviert ist und die Förderung der Schweizer Exportwirtschaft und die Erhaltung von Arbeitsplätzen in der Schweiz zum Ziel hat, mag dies weiter illustrieren. Dieses Ziel ist per Definition ein anderes als dasjenige der Entwicklungspolitik, welche das Wohl der Entwicklungsländer im 
Auge hat. Das Leitbild Nord-Süd strebt denn auch nicht eine vollständige Eliminierung der Widersprüche an, vielmehr ist sein Ziel, Interessenkonflikte darzulegen und transparent zu machen, damit die verschiedenen Belange der Akteure in die Entscheidungsfindung einbezogen werden können. Letzten Endes geht es dabei jedoch immer um eine politische Güterabwägung, um die Frage, mit welcher Gesamtpolitik in welchem Umfeld können mit dem kleinsten Eigenverzicht durch einen Kleinstaat wie die Schweiz die gesteckten Ziele erreicht werden. Eine Politik der Konfrontation dient letztlich niemandem, das Beispiel Hollands hat dies gezeigt. Mit einer allein auf die Wahrung der Menschenrechte ausgerichteten Haltung gegenüber Indonesien hat șich die holländische Regierung vom politischen Dialog mit Indonesien ausgeschlossen und hat dabei seine wirtschaftlichen Beziehungen riskiert, ohne dass damit der Menschenrechtssituation in Indonesien geholfen wurde. Eine solche Politik liesse uns den einzigen Vorteil verlieren, den wir als Kleinstaat mit einem guten Ruf haben, nämlich über wirtschaftliche Beziehungen und den politischen Dia$\log$ Einfluss auf das Geschehen und die Verhaltensweisen zu nehmen. Dieser Einfluss spielt übrigens auch eine wichtige Rolle in internationalen Gremien und Vereinbarungen, wo die Stimme der Schweiz als Kleinstaat, der keine Grossmachtinteressen verfolgt, immer wieder ein positives Echo findet.

Im Rahmen seiner Entwicklungspolitik entwickelt das Bundesamt für Aussenwirtschaft (BAWI) zur Zeit ein neues Instrumentarium, welches den Liberalisierungs- und Privatisierungsprozess in Entwicklungsländern gezielter und direkter unterstützen und Privatinvestitionen fördern soll. Ziel der neuen Instrumente ist es, den Transfer von Kapital, Technologie und Know-how in die Länder des Südens zu fördern. Dabei soll die Entwicklung von partnerschaftlicher Zusammenarbeit zwischen Unternehmen des Nordens und des Südens, welche über einfache Handelstransaktionen hinausgeht und eine faire Risikound Gewinnaufteilung einschliesst, unterstützt werden. Der Einbezug von Ressourcen und Expertise schweizerischer Unternehmen soll darin so weit wie möglich berücksichtigt werden.

\section{Mechanismen}

In unseren Augen sollte die Suche nicht so sehr neuen Mechanismen gelten, sondern mehr der Frage, wie bestehende Mechanismen besser genutzt, d.h. Kohärenzkriterien besser einbezogen werden können. In der Tat existieren bereits eine Reihe von institutionellen Mechanismen, welche mehr oder weniger erfolgreich versuchen, sich dieser Frage anzunehmen. Auf internationaler Ebene ist zum Beispiel die Konsultativgruppe Indonesien unter der Leitung der Weltbank zu erwähnen, die jährlich zusammentrifft, um mit den indonesischen Behörden politische und wirtschaftliche Fragen zu erörtern und die Aktivitäten der verschiedenen Geber zu koordinieren. Die Schweiz nimmt in diesem Forum eine aktive Rolle wahr. Über die schweizerische Mitgliedschaft in den Bretton 
Woods-Instituten und Regionalbanken ${ }^{2}$ nehmen wir regelmässig, z.T. in Konsultation mit Hilfswerken und Nichtregierungsorganisationen (NGO), Stellung zu Ländern, Bereichen und Einzelfragen, in denen wir entwicklungspolitisch schwerpunktmässig tätig sind. Nicht zu unterschätzen ist auch die Rolle der Diplomatie (Botschaft, Besuche), um einen konstanten Dialog aufrechtzuerhalten. In der achtköpfigen ERG-Kommission nimmt ein Vertreter der Direktion für Entwicklungszusammenarbeit und humanitäre Hilfe Einsitz, der die Aufgabe hat, die anderen Kommissionsmitglieder in Belangen der Entwicklungspolitik zu sensibilisieren. Daneben besteht ein Konsultationsmechanismus (Fragebogen) mit dem Entwicklungsdienst des BAWI bei ERG-Geschäften in ärmeren Entwicklungsländern. Bei grossen und/oder umstrittenen Projekten wird zudem die Weltbank konsultiert. Mit der Gründung der Wirtschaftskammer SchweizASEAN wird auch die Privatwirtschaft in der Region besser organisiert sein. Denkbar wäre auch ein Mitglieds- oder Beobachterstatus der in Indonesien tätigen NGO in dieser Kammer.

Wichtig erscheint vor allem ein verstärkter und gezielterer Informationsaustausch zwischen den einzelnen interessierten Kreisen. Die Organisation von Seminaren/Workshops zu aktuellen Themen könnte hier wahrscheinlich zu einem besseren Verständnis der einzelnen Interessengruppen und über lokale Mentalitäten und Belange beitragen.

Schliesslich möchten wir noch auf die Frage eingehen, ob sich die Entwicklungszusammenarbeit als Einflussmittel zur Erreichung einer höheren Kohärenz eignet. Am oben aufgeführten Beispiel Holland wird klar, dass die EZA von der Schweiz als kleines Land mit beschränkter Verhandlungsmacht sicher nicht als globales Druckmittel eingesetzt werden kann. Wenn jedoch die Erreichung der gesetzten Ziele nicht oder nicht mehr gewährleistet wird, d.h. wenn jegliche Beteiligung der Bevölkerung am Entwicklungsprozess ausgeschlossen ist, scheuen wir uns nicht, entsprechende Massnahmen zu ergreifen. Die oft vorgebrachte Alternative, bei Zweifeln z.B. an der Rechtmässigkeit an der Regierung eines Landes nur noch Basisprojekte zu unternehmen, ist unseres Erachtens fraglich, denn auch diese können strukturerhaltend wirken. Hingegen können auch traditionelle Enwicklungsprojekte einen "subversiven“ Einfluss haben, man denke z.B. an Projekte mit lokalen NGO oder Projekte, welche die Stärkung des institutionellen Umfelds zum Ziel haben. Auch wenn ihr Ziel rein technisch erscheint, beinhalten solche Projekte oft ein Änderung des institutionellen Rahmens und die Beteiligung der Bevölkerung, was sich gegen die etablierte Politik richten kann.

2. Interamerikanische Entwicklungsbank, Afrikanische Entwicklungsbank, Asiatische Entwicklungsbank 